\title{
Serous Endometrial Intraepithelial Carcinoma Involving Endometrial Polyp Diagnosed by Transcervical Resection: A Case Report
}

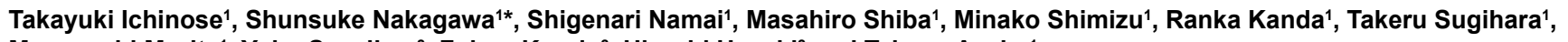
Masayoshi Morita', Yuko Sasajima², Fukuo Kondo², Hiroshi Uozaki ${ }^{2}$ and Takuya Ayabe ${ }^{1}$

${ }^{1}$ Department of Obstetrics and Gynecology, Graduate School of Medicine, Teikyo University, Tokyo, Japan ${ }^{2}$ Department of Pathology, Graduate School of Medicine, Teikyo University, Tokyo, Japan

\begin{abstract}
Uterine serous carcinoma is a representative histological subtype of endometrial type II cancer. Uterine serous intraepithelial carcinoma is thought to be a precursor lesion of uterine serous carcinoma. Uterine serous intraepithelial carcinoma is frequently found to involve an endometrial polyp. Preoperative diagnosis of uterine serous intraepithelial carcinoma is difficult due to its localized occurrence in the endometrial cavity. We experienced a case of postmenopausal women with abnormal endometrial cytology. Endometrial biopsy suggested the possibility that she has a malignant uterine tumor, but it did not reached to the confirmative pathological diagnosis. Hysteroscopy revealed that she has an endometrial polyp. Pathological analysis of the resected endometrial polyp confirmed that her disease is uterine serous intraepithelial carcinoma involving endometrial polyp. She underwent hysterectomy, bilateral salpingo-oophorectomy, omentectomy and retroperitoneal lymphadenectomy. No extrauterine lesion was detected. Our case suggested the possibility that transcervical resection under the hysteroscope might be useful to detect serous endometrial intraepithelial carcinoma.
\end{abstract}

Keywords: Endometrial cancer; Serous endometrial cancer; Endometrial intraepithelial cancer, Endometrial polyp; p53; Hysteroscopy; Transcervical resection

\section{Introduction}

Endometrial cancer is classified into two groups- type I and type II based on pathological characteristics, as well as molecular pathogenesis and clinical profiles [1]. The endometrial type I cancer is estrogendependent, of low-grade endometrioid histology and arises in the background of endometrial hyperplasia, its precursor lesion [1]. The type 1 cancer usually occurs in the pre and peri-menopausal women and strongly linked to obesity [1]. The endometrial type II cancer is estrogen-independent tumor with high-grade histology, i.e., high-grade endometrioid, serous and clear cell. It usually occurs in non-obese and post-menopausal elderly women. Uterine serous carcinoma is a major subtype of endometrial type II cancer. In contrast to endometrioid carcinoma, little is known about its cancer development process and precursor lesion. Minimal uterine serous carcinoma has been proposed to be a precursor lesion of uterine serous carcionoma. [2-4]. It has been proposed to include serous carcinomas with invasion limited to the superficial endometrium, and those without stromal invasion [3]. The latter entities have been classified as serous endometrial intraepithelial carcinoma [5-8]. Noninvasive USC is called Serous Endometrial Intraepithelial Carcinoma [SEIC], and USC with limited infiltration is called Superficial Serous Carcinoma [SSC]. SEIC is thought to be a precursor lesion of serous endometrial carcinoma [9]. Recent studies have been reported that most of uterine serous intraepithelial carcinoma cases occur in the endometrial polyp $[3,8,10]$. Ono et al., analyzed 6 cases of uterine superficial serous carcinomas or extensive serous endometrial intraepithelial carcinomas and reported that all but 1 patient had tumors originating from the surface of polyps, including 3 patients who each had an enormous polyp occupying the entire uterine cavity [10]. Recent study also described a case of serous endometrial intraepithelial carcinoma associated with submucosal leiomyomatous polyp [11].

Here, we report a case of serous intraepithelial carcinoma arising in the endometrial polyp, which was diagnosed by the transcervical resection under hysteroscopy.

\section{Case Report}

A 59-year-old gravida 2 Para 2 woman presented with postmeno- pausal bleeding. The transcervical ultrasonography detected her thickened endometrium. Her endometrial cytology suggested that she might have uterine malignancy. The endometrial biopsy also suggested the possibility of endometrial cancer, but it did not reached to the confirmative diagnosis. Magnetic resonance imaging showed endometrial polyp with about 1 centimeter in diameter in her uterine endometrial cavity. The hysteroscopy identified endometrial polyp arising from the anterior uterine wall (Figure 1). Her endometrium looked atrophic, except of a proliferated endometrial polyp. The endometrial polyp was resected by the transcervical resection under the hysteroscopy (Figures $1 \mathrm{a}$ and $1 \mathrm{~b})$. Pathological examination revealed that she has uterine serous intraepithelial carcinoma (Figures 2a). The immunohistochemical

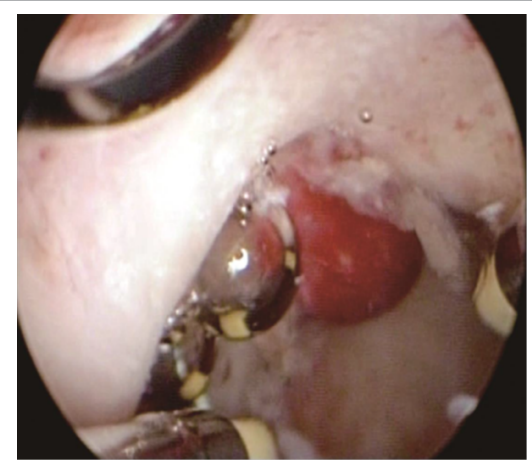

Figure 1a: Endometrial polyp had been developed from the anterior wall of uterus

*Corresponding author: Shunsuke Nakagawa, Department of Obstetrics and Gynecology, Graduate School of Medicine, Teikyo University, Tokyo, Japan, Tel: 81-3-3964-1211; Fax: 81-3-5373-1274; E-mail: shunsuke@med.teikyo-u.ac.jp

Received December 13, 2015; Accepted January 05, 2016; Published January 12, 2016

Citation: Ichinose T, Nakagawa S, Namai S, Shiba M, Shimizu M, et al. (2016) Serous Endometrial Intraepithelial Carcinoma Involving Endometrial Polyp Diagnosed by Transcervical Resection: A Case Report. J Clin Case Rep 5: 678. doi:10.4172/2165-7920.1000678

Copyright: ( 2016 Ichinose T, et al. This is an open-access article distributed under the terms of the Creative Commons Attribution License, which permits unrestricted use, distribution, and reproduction in any medium, provided the original author and source are credited. 


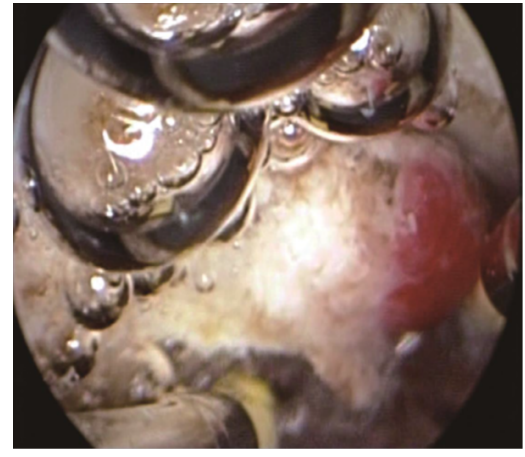

Figure 1b: Endometrial polyp resected by the transcervical resection by hysteroscopy.

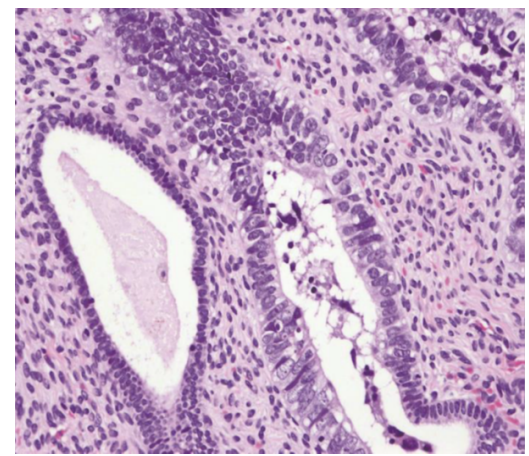

Figure 2a: Hematoxylin and eosin Staining of serous endometrial intraepithelial carcinoma.

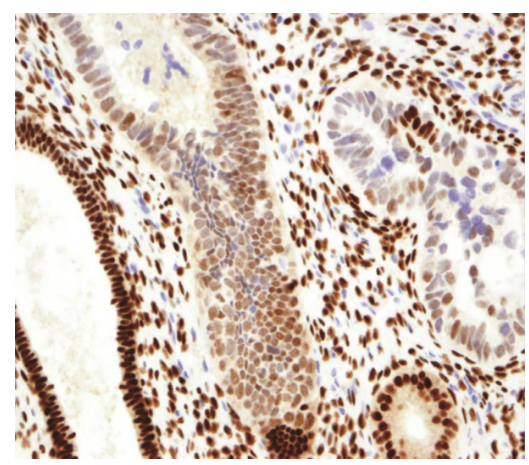

Figure 2b: Expression of Estrogen receptor.

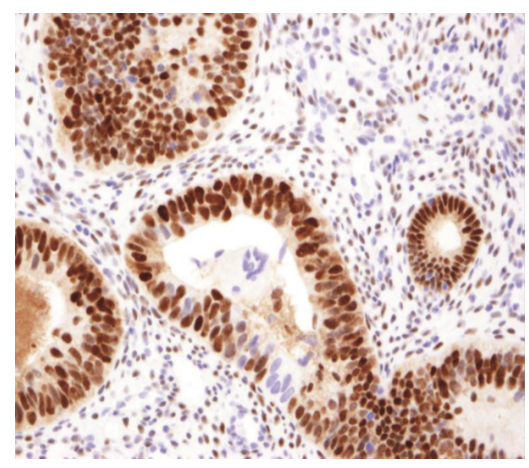

Figure 2c: Expression of Progesterone receptor. staining analysis showed that the uterine serous intraepithelial carcinoma is positive for estrogen receptor, progesterone receptor, HER2, and Cyclin E (Figures $2 \mathrm{~b}-2 \mathrm{f}$ ). The immunohistochemical staining of $\mathrm{p} 53$ was completely negative (Figure 2d). She underwent hysterectomy, bilateral salpingo-oophorectomy, omentectomy and retroperitoneal lymphadenectomy. The endometrium of the uterus showed atrophic pattern with a root of the endometrial polyp (Figure 3). No extrauterine lesion was detected. Six cycles of adjuvant chemotherapy were given.

\section{Discussion}

We experienced a case of serous endometrial intraepithelial carcinoma involving endometrial polyp diagnosed with transcervical resection. Minimal uterine serous carcinoma is found to involve an endometrial polyp in most cases $[3,8]$. There is no detailed case report showing the image under hysteroscopy of the disease, thus far. We presented the image of serous endometrial intraepithelial carcinoma involving endometrial polyp under the hysteroscopy and pathological findings of the disease.

The serous endometrial intraepithelial carcinoma has been regarded as in situ serous adenocarcinoma of the endometrium. It occurs in the atrophic and resting endometrium and frequently involves endometrial polyp $[3,8]$. The lesion of the serous endometrial intraepithelial carcinoma is limited to the focal area in the endometrium. The blind endometrial biopsy tends to be false negative. Our case showed that transcervical resection under hyspteroscope might to be a suitable tool to detect the disease.

The serous endometrial intraepithelial carcinoma has extrauterine tumor spread in most of diseases $[3,8,12,13]$. The omentum was frequently involved in most of cases. There was no extrauterine spread of disease in our case. Recent study showed that fallopian tube represents the primary lesion of some of serous endometrial carcinoma cases [9]. The detailed analysis of fallopian tube would shed light on multifocal carcinogenesis of serous intraepithelial carcinoma and serous endometrial carcionoma.

Most of serous endometrial intraepithelial carcinoma demonstrate a diffuse and overexpression of p53 [14]. The immunohistochemical analysis of p53 was completely negative in our case (Figure 2). That might suggest the possibility that serous endometrial intraepithelial carcinoma in our case might have a p53 alteration that results in a complete deletion of the gene or an early termination of its transcription. The previous study showed that $10 \%$ of intraepithelial carcinoma cases showed the null phenotype of p53 [15]. Our data regarding p53 null phenotype is in line with the previous study [15]. P53 alteration is a molecular signature of serous endometrial carcinoma. P53 mutational analysis is reported to be a useful tool to detect the primary source of extrauterine metastatic lesions of the serous intraepithelial carcinoma [16]. Additional molecular events might to be involved in carcinogenesis of serous endometrial carcinogenesis, even in endometrial intraepithelial carcinoma. Recent research reported that the following genes alterations in addition to p53 in uterine serous carcinoma were reported; PIK3CA [29\%], FBXW7 [12\%], KRAS [9.3\%], BRCA1 [9.1\%] and BRCA2 [6.3\%] [17]. Some of uterine serous carcinomas are reported to associate with germline mutation of BRACA1 [18]. Mutational analysis of these genes in the serous intraepithelial carcinoma will reveal the molecular carcinogenesis steps in the development of serous endometrial intraepithelial carcinoma and serous endometrial carcinoma. Our analysis suggests the possibility that HER2 and Cyclin-E overexpressions might have a role in its carcinogenesis. Recent study showed that neratinib, the small tyrosine 


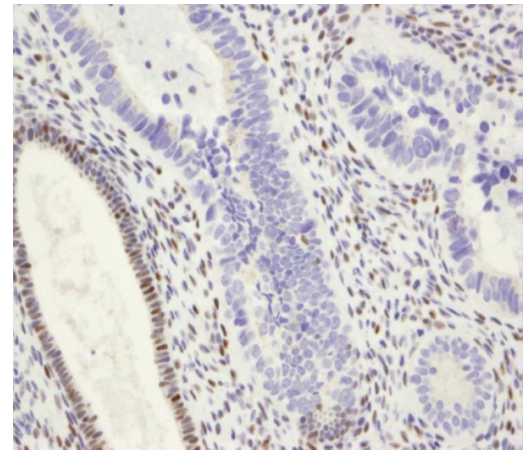

Figure 2d: p53 mutation[null pattern].

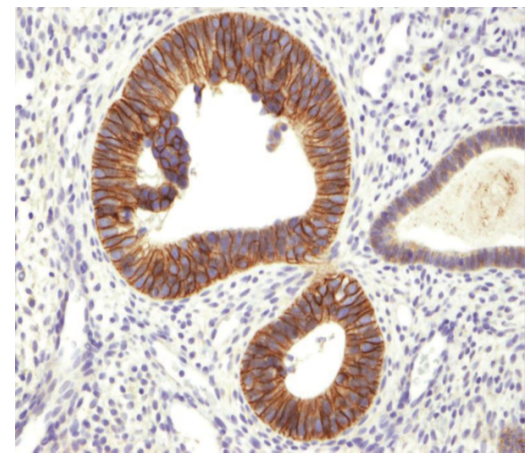

Figure 2e: Expression of HER2.

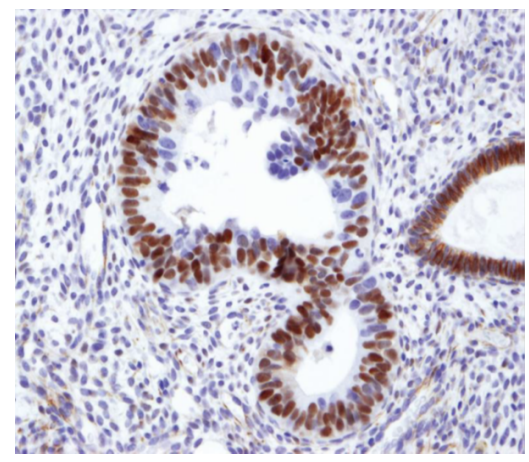

Figure 2f: Expression of cyclin E.

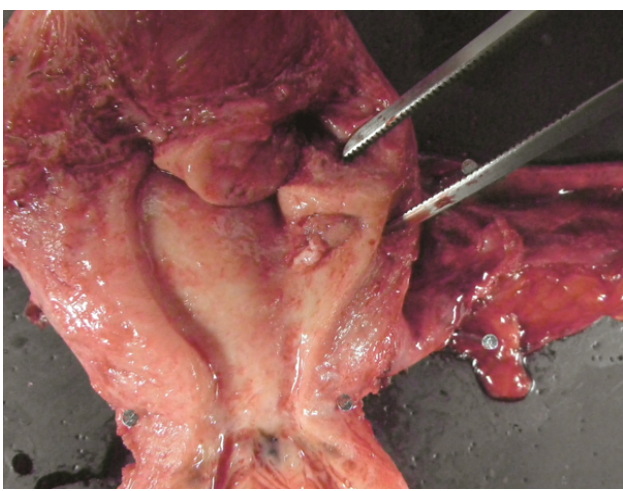

Figure 3: The gross appearance of the uterus. The arrow indicates the root of endometrial polyp. kinase inhibitor against ErbB1 and HER2, might be a potential treatment option for patients harboring HER2/neu amplified uterine serous carcinoma. Our case suggested the possibility that transcervical resection under the hysteroscopy is useful to detect serous endometrial intraepithelial carcinoma, which is focal lesion in uterine endometrial cavity.

\section{References}

1. Bokhman JV (1983) Two pathogenetic types of endometrial carcinoma. Gynecol Oncol 15: 10-17.

2. Rabban JT, Zaloudek CJ (2007) Minimal uterine serous carcinoma: current concepts in diagnosis and prognosis. Pathology 39: 125-133.

3. Hui P, Kelly M, O'Malley DM, Tavassoli F, Schwartz PE (2005) Minimal uterine serous carcinoma: a clinicopathological study of 40 cases. Mod Pathol 18: 75 82 .

4. Wheeler DT, Bell KA, Kurman RJ, Sherman ME (2000) Minimal uterine serous carcinoma: diagnosis and clinicopathologic correlation. Am J Surg Pathol 24: 797-806.

5. Ambros RA, Sherman ME, Zahn CM, Bitterman P, Kurman RJ (1995) Endometrial intraepithelial carcinoma: a distinctive lesion specifically associated with tumors displaying serous differentiation. Hum Pathol 26: 1260-1267.

6. Maksem JA, Lee SS (1998) Endometrial intraepithelial carcinoma diagnosed by brush cytology and p53 immunostaining, and confirmed by hysterectomy. Diagn Cytopathol 19: 284-287.

7. Pathiraja P, Dhar S, Haldar K (2013) Serous endometrial intraepithelial carcinoma: a case series and literature review. Cancer Manag Res 5: 117-122.

8. Yasuda M, Katoh T, Hori S, Suzuki K, Ohno K, et al. (2013) Endometrial intraepithelial carcinoma in association with polyp: review of eight cases. Diagn Pathol 8: 25.

9. Tolcher MC, Swisher EM, Medeiros F, Lima JF, Hilderbrand JL, et al. (2015) Characterization of precursor lesions in the endometrium and fallopian tube epithelium of early-stage uterine serous carcinoma. Int J Gynecol Pathol 34: $57-64$

10. Ono K, Hayashi H, Tateno M, Tanaka R, Suzuki R, et al. (2014) Uterine superficial serous carcinomas and extensive serous endometrial intraepithelial carcinomas: clinicopathological analysis of 6 patients. Int J Clin Exp Pathol 7: 7979-7988.

11. Yang M, Assylbekova B (2015) Serous endometrial intraepithelial carcinoma associated with submucosal leiomyomatous polyp: The first reported case. J Obstet Gynaecol 3: 533-535.

12. Hou JY, McAndrew TC, Goldberg GL, Whitney K, Shahabi S (2014) A clinica and pathologic comparison between stage-matched endometrial intraepithelia carcinoma and uterine serous carcinoma: is there a difference? Reprod Sci 21: 532-537.

13. Maenaka T, Egawa-Takata T, Hisamoto K, Kunishige I, Nishio Y, et al. (2014) Case of uterine papillary serous carcinoma following tamoxifen treatment that could not be diagnosed during screening. J Obstet Gynaecol Res 40: 14501454.

14. Kovalev S, Marchenko ND, Gugliotta BG, Chalas E, Chumas J, et al. (1998) Loss of p53 function in uterine papillary serous carcinoma. Hum Pathol 29: 613-619.

15. Nafisi H, Ghorab Z, Ismill N, Dubé V, Plotkin A, et al. (2015) Immunophenotypic Analysis in Early Müllerian Serous Carcinogenesis. Int J Gynecol Pathol 34: 424-436.

16. Jia L, Yuan Z, Wang Y, Cragun JM, Kong B, et al. (2015) Primary sources of pelvic serous cancer in patients with endometrial intraepithelial carcinoma. Mod Pathol 28: 118-127.

17. Jones NL, Xiu J, Reddy SK, Burke WM, Tergas AI, et al. (2015) Identification of potential therapeutic targets by molecular profiling of 628 cases of uterine serous carcinoma. Gynecol Oncol 138:620-6.

18. Hecht JL, Konstantinopoulos PA, Awtrey CS, Soslow RA (2014) Immunohistochemical loss of BRCA1 protein in uterine serous carcinoma. Int $J$ Gynecol Pathol 33: 282-287. 